\title{
Selected Parameters of Venous Blood Gas Analysis in Brachy- cephalic Dogs with Brachycephalic Obstructive Airway Syn- drome before and after Surgical Treatment
}

\author{
Erjavec $\mathbf{V}^{1}$, Nemec Svete $\mathbf{A}^{1, *}$ \\ 1. University of Ljubljana, Veterinary Faculty, Small Animal Clinic, Ljubljana, Slovenia \\ * Correspondence: Alenka Nemec Svete; alenka.nemecsvete@vf.uni-lj.si
}

Citation: Erjavec V, Nemec Svete A. Selected parameters of venous blood gas analysis in brachycephalic dogs with brachycephalic obstructive airway syndrome before and after surgical treatment. Proceedings of Socratic Lectures. 2021; 6: 1-6. https://doi.org/10.55295/PSL.2021.D. 001

Publisher's Note: UL ZF stays neutral with regard to jurisdictional claims in published maps and institutional affiliations.

Copyright: (c) 2021 by the authors. Submitted for possible open access publication under the terms and conditions of the Creative Commons Attribution (CC BY) license (https://creativecommons.org/license s/by/4.0/).

\begin{abstract}
:
Brachycephalic dog breeds, such as Pug, English and French Bulldogs and Boston Terrier belong to a group of breeds characterized by a severe shortening of the muzzle and thus the underlying bones, as well as a more modest shortening and widening of the skull. These breeds are prone to a conformation-related disorder known as brachycephalic obstructive airway syndrome (BOAS). Among consequences of the upper airway abnormalities is not only hypoxia, but also redued clearance of carbon dioxide. The aim of the present study was to evaluate selected parameters $\left(\mathrm{pH}_{\text {, }}\right.$ partial pressure of carbon dioxide, bicarbonate concentration and base excess) of venous blood gas analysis in brachycephalic dogs with BOAS before and one month after surgical treatment of BOAS. Sixty-two BOAS patients with different severity grades of BOAS were enrolled in the study. Patients were classified as grade 1, grade 2, and grade 3 based on the decrease in the radius of the airway at the level of the nasopharynx, oropharynx, laryngopharynx, and larynx after soft palate surgery. Contrary to our expectations, surgical treatment had no effect on selected venous blood gas parameters in any of the grades of BOAS. However, selected venous blood gas parameters deviated from the normal range before and after surgical treatment, indicating respiratory acidosis in our BOAS patients. Based on the results we may conclude that venous blood gas analysis could help in assessing the health status of brachycephalic dogs with BOAS.
\end{abstract}

Keywords: Brachycephaly; Brachycephalic obstructive airway syndrome; Dogs; Respiratory acidosis; Surgery; Venous blood gas analysis 


\section{Introduction}

Brachycephalic dog breeds, including Pug, English and French Bulldogs and Boston Terrier belong to a group of breeds characterized by a severe shortening of the muzzle and thus the underlying bones, as well as a more modest shortening and widening of the skull. In recent years, brachycephalic breeds have become increasingly popular companion dog breeds. Their popularity may be due to the similarity between the head shape of brachycephalic dogs and that of human infants. However, brachycephalic breeds have been shown to be generally less healthy than non-brachycephalic breeds and have shorter lifespan (Fawcett et al., 2018; Packer et al., 2019; O'Neill et al., 2020). Artificial selection for extreme brachycephaly has resulted in deformation of the upper airway tract leading to obstruction as the soft tissues have not shortened in proportion to the length of the skull. Brachycephalic dogs are prone to a conformation-related disorder known as brachycephalic obstructive airway syndrome (BOAS) (Packer et al., 2015; Liu et al., 2017; Fawcett et al., 2018; Packer et al., 2019). Clinical signs of BOAS include inspiratory dyspnoea, snoring, stertor and stridor, panting, stress, exercise and heat intolerance, cyanosis, and even syncopal episodes, gastrointestinal problems, and disturbed sleep patterns (Meola, 2013; Roedler et al., 2013; Dupre and Heidenreich, 2016; Packer et al., 2019). Treatment of affected dogs can be surgical or conservative, with the latter including medical treatment, weight management, and keeping patients cool, calm and with low physical activity (Torrez and Hunt, 2010; Meola, 2013; Dupre and Heidenreich, 2016). However, conservative treatment can only temporarily improve clinical signs and is thus merely palliative. Surgical treatment, which includes widening of the stenotic nares, staphylectomy and, in selected cases, ventriculectomy (sacculectomy) to improve airflow through the rima glottidis, is therefore recommended in most BOAS-affected dogs (Torrez and Hunt, 2010; Dupre and Heidenreich, 2016). Brachycephalic dogs have additional systemic complications (Meola, 2013; Mellema and Hoareau, 2014; Fawcett et al., 2018; O'Neill et al., 2020). Among consequences of the BOAS is not only hypoxia, but also redued clearance of carbon dioxide. Systemically healthy brachycephalic dogs have been found to have hypertension and significantly higher arterial partial pressure of carbon dioxide $\left(\mathrm{PaCO}_{2}\right)$ and significantly lower arterial partial pressure of oxygen $\left(\mathrm{PaO}_{2}\right)$ and arterial haemoglobin oxygen saturation $\left(\mathrm{SaO}_{2}\right)$ compared to non-brachycephalic breeds (Hoareau et al., 2012; Arulpagasam et al., 2018). Significantly lower $\mathrm{SaO}_{2}$ (Dias et al., 2016; Arulpagasam et al., 2018) and $\mathrm{PaO}_{2}$ (Canola et al., 2018) were reported in brachycephalic dogs with BOAS compared to non-brachycephalic dogs. On the other hand, no significant difference in arterial $\mathrm{pH}$, $\mathrm{PaO}_{2}$, bicarbonate $\left(\mathrm{HCO}_{3}{ }^{-}\right)$concentration and $\mathrm{PaCO}_{2}$ was reported between BOAS-affected dogs and non-brachycephalic dogs (Dias et al., 2016; Arulpagasam et al., 2018). Arterial blood gas analysis is the gold standard for evaluating hypoxemia in patients with respiratory disorders. Peripheral venous blood gas analysis has been proposed as an alternative to arterial blood gas analysis for hypercapnia and metabolic disturbances, such as acidosis (Byrne et al., 2013). Nowadays, the venous blood gas analysis is increasingly used by veterinary practitioners, thus providing important information regarding acid-base status and ventilation (Ilkiw et al., 1991). Arterial blood sampling in conscious BOAS patients is risky due to their breathing difficulties resulting from upper airway obstruction (Hendricks, 1992; Arulpagasam et al., 2018). In BOAS patients, both arterial and venous blood gas analysis can be useful. If an arterial blood sample cannot be obtained, a venous blood sample may provide useful information about $\mathrm{pH}, \mathrm{HCO}_{3}{ }^{-}$and $\mathrm{PCO}_{2}$ levels in these patients (Hendricks, 1992). To the authors' knowledge, only three studies have been published reporting results of arterial blood gas analysis in BOAS patients (Dias et al., 2016; Arulpagasam et al., 2018; Canola et al., 2018), but there are none reporting results of venous blood gas analysis in these patients. Therefore, the aim of this study was to evaluate selected parameters of venous blood gas analysis $\left(\mathrm{pH}, \mathrm{PCO}_{2}, \mathrm{HCO}_{3}\right.$, base excess $\left.(\mathrm{BE})\right)$ in brachycephalic dogs with different severity of BOAS before and after surgical treatment of BOAS. 


\section{Materials and Methods}

\subsection{Dogs}

A total of 62 client-owned dogs diagnosed with BOAS were included in the study. At the time of initial presentation, the history of the dogs was collected using a questionnaire about behaviour, health, and lifestyle. All the dogs that had any signs of concurrent disease or received any therapy or vaccination within the previous month were excluded from the study. Brachycephalic obstructive airway syndrome diagnosis was carried out based on clinical signs of upper airway obstruction and anatomical abnormalities, as has been described elsewhere (Dupre and Heidenreich, 2016; MacPhail and Fossum, 2019). Patients were classified as grade 1, grade 2, and grade 3 based on the decrease in the radius of the airway at the level of the nasopharynx, oropharynx, laryngopharynx, and larynx after soft palate surgery. Grade 1 patients had no or very mild narrowing of the airways, grade 2 patients had $50 \%$ decrease in airways radius and grade 3 patients had almost complete airway obstruction at the level of one or more of the following anatomic regions: nasopharynx, oropharynx, laryngopharynx and/or larynx. The patients' health status was assessed by history, physical examination, and blood tests including complete blood count with white blood cell differential count (data not shown), serum biochemical analyses (data not shown), and venous blood gas analyses (only selected parameters presented in the manuscript). The patients with BOAS were scheduled for surgical treatment under general anaesthesia. Stenotic nares were enlarged surgically by rhinoplasty. A horizontal wedge excision technique was used for excising part of ala nasi with a No. 11 scalpel blade. To oppose the wedge margins two to four simple interrupted sutures using synthetic absorbable monofilament suture material Glycomer 631 (Biosyn $4 / 0$, Covidien, Dublin, Ireland) were placed. To correct both the excessive length and excessive thickness of the soft palate, folded flap palatoplasty was performed in all dogs. A portion of oropharyngeal mucosa and underlying soft tissue was excised using a Surgitron Radiolase II (Ellman International, Inc., Hicksville, NY, USA). The formal written consent of the owner was obtained before the dogs entered the study. All procedures complied with the relevant Slovenian governmental regulations (Animal Protection Act UL RS, 43/2007).

\subsection{Blood collection and blood gas analysis}

Venous blood samples were collected twice from BOAS patients via the central venous catheter, 2 hours ( \pm 15 minutes) before premedication and oxygenation (before surgical treatment) and one month after surgical treatment. Blood samples for venous blood gas analyses were collected into blood gas syringes containing lithium heparin (Sarstedt, Numbrecht, Germany) and analysed immediately (less than 5 minutes) after collection using a blood gas analyser (RAPIDPoint 500, Siemens, Munich, Germany). The $\mathrm{pH}$ and $\mathrm{PCO}_{2}$ were corrected for rectally measured body temperature.

\subsection{Statistical analysis}

Data were analysed using commercial software (IBM SPSS 25.0, Chicago, Illinois, USA). The Shapiro-Wilk test was performed to test whether the data were normally distributed. According to the results of normality tests, parametric (one-way ANOVA with Tukey Honestly Significant Difference post hoc test; $\mathrm{pH}, \mathrm{PCO}_{2}, \mathrm{HCO}_{3}-\mathrm{BE}$ ) or non-parametric (Kruskal-Wallis test followed by multiple comparisons and Bonferroni correction; age, weight, body condition score (BCS)) tests were used to compare the parameters between the dog groups. Paired t-test was used to compare selected parameters of venous blood gas analysis $\left(\mathrm{pH}, \mathrm{PCO}_{2}, \mathrm{HCO}_{3}{ }^{-}, \mathrm{BE}\right)$ before and after surgery. The significance level was set at $5 \%$. 


\section{Results}

Selected parameters of venous blood gas analysis were evaluated before and after surgical treatment in 62 BOAS patients classified into grade 1 (10 dogs), grade 2 ( $26 \mathrm{dogs}$ ), and grade 3 (26 dogs) (Table 1). Patients in grade 1 were the youngest; however, we found no significant difference in age, nor in body weight and BCS between the groups of BOAS patients. French Bulldogs were the most common breed. These dogs accounted for 31 of the 62 cases.

Table 1. Baseline characteristics of brachycephalic dogs in different grades of brachycephalic obstructive airway syndrome (BOAS).

\begin{tabular}{|l|l|l|l|l|}
\hline & Grade 1 & Grade 2 & Grade 3 & All BOAS patients \\
\hline Number & 10 & 26 & 26 & 62 \\
\hline Sex (female/male) & $3 / 7$ & $12 / 14$ & $8 / 18$ & $23 / 39$ \\
\hline $\begin{array}{l}\text { Age (years) } \\
\text { Median } \\
\text { IQR }\end{array}$ & 1.29 & 2.54 & 3.04 & 2.58 \\
\hline $\begin{array}{l}\text { Weight (kg) } \\
\text { Median }\end{array}$ & $0.73-3.60$ & $1.33-3.71$ & $1.65-5.75$ & $1.40-4.75$ \\
IQR & 8.2 & 10.0 & 10.1 & 10.0 \\
\hline $\begin{array}{l}\text { BCS } \\
\text { Median }\end{array}$ & $5.8-12.3$ & $8.7-12.4$ & $8.4-11.8$ & $8.3-12.1$ \\
IQR & 3.0 & 3.0 & 3.0 & 3.0 \\
\hline Breeds & $3.0-3.0$ & $\begin{array}{l}13 \mathrm{FB}, 6 \mathrm{P}, \\
5 \mathrm{BST}, 1 \mathrm{~EB}, 1 \mathrm{ST}\end{array}$ & $\begin{array}{l}14 \mathrm{FB}, 6 \mathrm{P}, \\
5 \mathrm{BST}, 1 \mathrm{ST}\end{array}$ & $\begin{array}{l}3.0-4.0 \\
11 \mathrm{FB}, 12 \mathrm{P},\end{array}$ \\
\hline
\end{tabular}

$\mathrm{IQR}=$ interquartile range $\left(25^{\text {th }}\right.$ to $75^{\text {th }}$ percentile); BCS=body condition score; $\mathrm{EB}=$ English Bulldog; FB=French Bulldog; $\mathrm{P}=\mathrm{Pug}$; BST=Boston Terrier; ST=Shih-Tzu.

Selected parameters of venous blood gas analysis before and after surgery, as well as the corresponding reference intervals established in canine venous blood samples (Bachmann, et al. 2018) using the same analyser as in our study, are shown in Table 2. We found no significant difference in any of the selected blood gas parameters between the different grades of BOAS before and after surgical treatment.

Table 2: Selected venous blood gas parameters (mean \pm standard deviation) of brachycephalic dogs in different grades of brachycephalic obstructive airway syndrome (BOAS) before and after surgical treatment.

\begin{tabular}{|l|l|l|l|l|}
\hline & $\mathrm{pH}$ & $\begin{array}{l}\mathrm{PCO}_{2} \\
(\mathrm{mmHg})\end{array}$ & $\begin{array}{l}\mathrm{HCO}_{3^{-}} \\
(\mathrm{mmol} / \mathrm{L})\end{array}$ & $\begin{array}{l}\mathrm{BE}(\mathrm{ecf}) \\
(\mathrm{mmol} / \mathrm{L})\end{array}$ \\
\hline $\begin{array}{l}\text { Grade } 1 \\
\text { Before } \\
\text { After }\end{array}$ & $7.342 \pm 0.053$ & $44.26 \pm 7.54$ & $22.74 \pm 1.43$ & $-2.51 \pm 1.23$ \\
\hline $\begin{array}{l}\text { Grade } 2 \\
\text { Before } \\
\text { After }\end{array}$ & $7.337 \pm 0.523$ & $44.97 \pm 6.97$ & $22.91 \pm 1.33$ & $-2.48 \pm 1.16$ \\
\hline $\begin{array}{l}\text { Grade } 3 \\
\text { Before } \\
\text { After }\end{array}$ & $7.336 \pm 0.037$ & $43.57 \pm 5.85$ & $22.84 \pm 2.23$ & $-2.61 \pm 2.61$ \\
\hline REF & $7.323 \pm 0.066$ & $47.90 \pm 9.18$ & $22.59 \pm 2.09$ & $-2.94 \pm 2.16$ \\
\hline
\end{tabular}

$\mathrm{REF}=$ reference interval (Bachmann et al., 2018); $\mathrm{PCO}_{2}=$ partial pressure of carbon dioxide; $\mathrm{HCO}_{3}=$ bicarbonate concentration; $\mathrm{BE}$ (ecf)=base excess in extracellular fluid 


\section{Discussion}

The present study is the first that reports selected parameters of venous blood gas analysis in brachycephylic dogs with different grades of BOAS before and one month after surgical treatment. Therefore, our results were compared only to the published reference values of venous blood gas parameters determined in healthy dogs (Bachmann et al., 2018).

Although expected, we found no significant difference in any of the selected blood gas parameters when comparing them before and one month after surgical treatment in any of the groups of BOAS patients. These results suggest that surgical treatment had no effect on selected venous blood gas parameters or that the effect may not have been apparent because of the too short time after surgery. It may be that it would be more useful to take the blood samples six months after surgery. The mean values of $\mathrm{pH}$ were below the lower limit of the reference range (Bachmann et al., 2018) before and after surgical treatment in all groups of BOAS patients, indicating acidosis. Furthermore, the mean values of $\mathrm{PCO}_{2}$ exceeded the upper value of the reference range (Bachmann et al., 2018) in grade 3 of BOAS before and after surgical treatment and in grade 1 after surgery, indicating hypercapnia. High $\mathrm{PCO}_{2}$ values obtained in BOAS patients could be due to hypoventilation in these dogs as a result of upper airway obstruction. The results of our study indicate the presence of respiratory acidosis in our BOAS patients. As reported by Hendricks (1992), chronic respiratory acidosis could be present in BOAS. On the other hand, the mean values of bicarbonate and $\mathrm{BE}$, a quantity reflecting only the non-respiratory (metabolic) component of acid-base disturbances, remained within the reference intervals in all groups of BOAS patients before and after surgery. Based on our results, we may summarize that the changes in selected venous blood gas parameters found in our BOAS patients are indicative of respiratory acidosis and that these parameters are not affected by surgical treatment of BOAS. In addition to routine haematological and biochemical analyses, venous blood gas analysis could help in assessing the health status of brachycephalic dogs with BOAS. It is known that there are breed-specific anatomical airway differences between different brachycephalic breeds such as pugs and bulldogs, so the response to surgery can be unpredictable and breed-specific. Pugs have a significantly smaller rima glottidis than French Bulldogs, 82\% of them have nasopharyngeal turbinates, they also have a higher prevalence of laryngeal collapse $(96 \%)$, all of which may negate the surgical benefit. Therefore, further studies with a larger number of dogs are needed to evaluate the effect of breed on venous blood gas parameters and to assess whether surgical treatment of BOAS affects venous blood gas parameters in individual brachycephalic breeds.

\section{Conclusions}

Surgical treatment of BOAS had no effect on selected venous blood gas parameters in any of the grades of BOAS one month after surgery. Selected venous blood gas parameters deviated from the normal range before and after surgical treatment, indicating respiratory acidosis in our BOAS patients. Venous blood gas analysis could help in assessing the health status of brachycephalic dogs with BOAS.

Funding: This research was supported by Slovenian Research Agency (research program No. P4-0053).

Institutional Review Board Statement: All procedures complied with the relevant Slovenian governmental regulations (Animal Protection Act UL RS, 43/2007).

Conflicts of Interest: The authors declare no conflict of interest.

Acknowledgements: The authors thank Rebeka Turk and Luka Šparaš for the processing of the collected blood samples. 


\section{References}

1. Arulpagasam S, Lux C, Odunayo A, et al. Evaluation of pulse oximetry in healthy brachycephalic dogs. J Am Anim Hosp Assoc. 2018; 54: 344-350. DOI 10.5326/JAAHA-MS-6654

2. Bachmann K, Kutter APN, Jud Schefer R, Sigrist NE. Determination of reference intervals and comparison of venous blood gas parameters using a standard and nonstandard collection method in 51 dogs. SAT. 2018; 160: 163-170. DOI: $\underline{10.17236 / \text { sat00150 }}$

3. Byrne AL, Bennett M, Pace NL, Thomas P. Peripheral venous blood gas analysis versus arterial blood gas analysis for the diagnosis of respiratory failure and metabolic disturbances in adults (Protocol). Cochrane Database Syst Rev. 2013; 11: CD010841. DOI: 10.1002/14651858.CD010841

4. Canola RAM, Sousa MG, Braz JB, et al. Cardiorespiratory evaluation of brachycephalic syndrome in dogs. Pesq Vet Bras. 2018; 38: 1130-1136. DOI: 10.1590/1678-5150-PVB-5376

5. Dias MLM, Morris CFM, Moreti BM, et al. Anatomical, Cardiovascular, and Blood Gas Parameters in Dogs with Brachycephalic Syndrome. Acta Sci Vet. 2016; 44: 1356. DOI: https://doi.org/10.22456/1679-9216.80932

6. $\quad$ Dupre G, Heidenreich D. Brachycephalic Syndrome. Vet Clin Small Anim. 2016; 46: 691-707. https://doi.org/10.1016/j.cvsm.2016.02.002

7. Fawcett A, Barrs V, Awad M, et al. Consequences and Management of Canine Brachycephaly in Veterinary Practice: Perspectives from Australian Veterinarians and Veterinary Specialists. Animals (Basel). 2019; 9. DOI: 10.3390/ani9010003

8. Hendricks JC. Brachycephalic airway syndrome. Vet Clin North Am Small Anim Pract. 1992; 22: 1145-1153. DOI:10.1016/s0195-5616(92)50306-0

9. Hoareau GL, Jourdan G, Mellema M, Verwaerde P. Evaluation of arterial blood gases and arterial blood pressures in brachycephalic dogs. J Vet Intern Med. 2012; 26: 897-904. DOI: 10.1111/j.1939-1676.2012.00941.x

10. Ilkiw JE, Rose RJ, Martin IC. A comparison of simultaneously collected arterial, mixed venous, jugular venous and cephalic venous blood samples in the assessment of blood-gas and acid-base status in dog. J Vet Intern Med. 1991; 5: $294-298$. DOI: 10.1111/j.1939-1676.1991.tb03136.x

11. Liu NC, Troconis EL, Kalmar L, et al. Conformational risk factors of brachycephalic obstructive airway syndrome (BOAS) in pugs, French bulldogs, and bulldogs. PLoS One. 2017; 12: e0181928. DOI: 10.1371/journal.pone.0181928

12. MacPhail C, Fossum TW, Surgery of the upper respiratory system. In: Fossum TW, editor. Small animal surgery, $5^{\text {th }}$ edn. Philidelphia, Pennsylvania, Elsevier. 2019; pp. 833-883.

13. Mellema MS, Hoareau GL. Brachycephalic syndrome. In: Silverstein DC, Hopper K, editors. Small animal critical care medicine, $2^{\text {nd }}$ edn. St. Louis, Missouri, Elsevier. 2003; pp. 104-106.

14. Meola SD. Brachycephalic airway syndrome. Top Companion Anim Med. 2013; 28: 91-96. DOI: 10.1053/j.tcam.2013.06.004

15. O'Neill DG, Jackson C, Guy JH, et al. Epidemiological associations between brachycephaly and upper respiratory tract disorders in dogs attending veterinary practices in England. Canine Genet Epidemiol. 2015; 2: 10. DOI: $10.1186 / \mathrm{s} 40575-015-0023-8$

16. O'Neill DG, Pegram C, Crocker P, et al. Unravelling the health status of brachycephalic dogs in UK using multivariable analysis. Sci Rep. 2020; 10: 17251. DOI: 10.1038/s41598-020-73088-y

17. Packer RMA, Hendricks A, Tivers MA, Burn CC. Impact of facial conformation on canine cealth: Brachycephalic Obstructive Airway Syndrome. PLoS One. 2015; 10: e0137496. DOI: 10.1371/journal.pone.0137496

18. Packer RMA, O'Neill DG, Fletcher F, Farnworth MJ. Great expectations, inconvenient truths, and the paradoxes of the dog-owner relationship for owners of brachycephalic dogs. Plos One. 2019; 14: e0219918. DOI: 10.1371/journal.pone.0219918

19. Roedler FS, Pohl S, Oechtering GU. How does severe brachycephaly affect dog's lives? Results of a structured preoperative owner questionnaire. Vet J. 2013; 198: 606-610. DOI: 10.1016/j.tvjl.2013.09.009

20. Torrez CV, Hunt GB. Results of surgical correction of abnormalities associated withbrachycephalic airway obstruction syndrome in dogs in Australia. J Small Anim Pract. 2006; 47: 150-154. DOI: 10.1111/j.1748-5827.2006.00059.x 